\title{
Generation of electron bunches at low repetition rates using a beat-frequency technique
}

\author{
M. Poelker, J. Grames, J. Hansknecht, R. Kazimi, and J. Musson \\ Thomas Jefferson National Accelerator Facility, 12000 Jefferson Avenue, Newport News, Virginia 23606, USA
}

(Received 28 February 2007; published 16 May 2007)

\begin{abstract}
Even at a continuous wave facility such as CEBAF at Jefferson Lab, an electron beam with long time intervals (tens of ns) between individual bunches can be useful, for example, to isolate sources of background via time of flight detection or to measure the energy of neutral particles that cannot be separated with a magnetic field. This paper describes a demonstrated method to quickly and easily deliver bunches with repetition rates of 20 to $100 \mathrm{MHz}$ corresponding to time intervals between 50 and $10 \mathrm{~ns}$ (respectively). This is accomplished by changing the ON/OFF frequency of the photogun drive laser by a small amount $(\Delta f / f<20 \%)$, resulting in a bunch frequency equal to the beat frequency between the radio frequencies of the drive laser and the photoinjector chopper system.
\end{abstract}

DOI: 10.1103/PhysRevSTAB.10.053502

PACS numbers: 29.27.Eg, 29.27.Ac, 29.25.Bx, 29.27.Hj

\section{INTRODUCTION}

A DC high-voltage GaAs photogun [1] provides the electrons at the Continuous Electron Beam Accelerator Facility (CEBAF) [2] at Jefferson Lab. Under normal operating conditions, three drive lasers, one for each experimental hall, emit $\sim 40$ ps (FWHM) optical pulses with $499 \mathrm{MHz}$ pulse repetition rate corresponding to the third subharmonic of the CEBAF accelerating cavities. The light from these lasers is combined and directed at the same location on a GaAs photocathode within the $100 \mathrm{kV} \mathrm{DC}$ high-voltage photogun to produce three independent electron pulse trains interleaved in time and each experimental hall receives an electron beam with $2 \mathrm{~ns}$ bunch spacing.

For some nuclear physics experiments, however, it is desirable to operate with larger bunch spacing. For example, the $\mathrm{G}^{0}$ forward angle experiment [3] measured parity violating asymmetries in elastic electron-proton scattering. An electron beam with 32 ns bunch spacing was required to help distinguish elastically scattered protons from "slower" low energy background composed of pions and inelastically scattered protons. It took hours to install and remove the large modelocked laser used to produce the necessary time structure. In this paper, a new technique is described to quickly create a low repetition rate beam with large time intervals between bunches using the same lasers that normally operate at $499 \mathrm{MHz}$. This is accomplished by operating the drive laser at a pulse repetition rate only slightly different from the normal operating frequency. The experimental hall receives beam at the beat frequency between the drive laser and the photoinjector chopper. Repetition rates from 20 to $100 \mathrm{MHz}$ were demonstrated, with bunch separation ranging from 50 to $10 \mathrm{~ns}$. This beat-frequency technique is considerably easier to implement compared to installing a completely new laser, and although it provides only a few microamperes of low duty factor beam, we expect it will become a valuable and widely used tool for nuclear physics experiments at CEBAF.

\section{EXPERIMENT}

As mentioned above, CEBAF photogun drive lasers normally create electron beams with a $499 \mathrm{MHz}$ pulse repetition rate corresponding to $2 \mathrm{~ns}$ bunch spacing. The photoinjector passes $100 \mathrm{keV}$ electrons within a $20^{\circ}$ phase window at $499 \mathrm{MHz}$. Electrons outside this window are dumped within an rf chopper system composed of two $499 \mathrm{MHz} \mathrm{TM}_{210}$ mode deflecting cavities [4] (Fig. 1, top). The first cavity sweeps the electron beam along a circular path and the second cavity removes the kick, restoring the original trajectory. Solenoid magnets image the beam from one rf cavity to the next. The phase of the drive laser is adjusted to vary the arrival time of each electron pulse train at the chopper. For the proper laser phase, nearly all of the electrons within the $499 \mathrm{MHz}$ pulse train pass through an aperture in a water-cooled copper plate midway between the two rf cavities. To accommodate simultaneous experiments at multiple halls, there are three independent drive lasers that produce interleaved $499 \mathrm{MHz}$ pulse trains, and three chopper apertures with variable width, that can be independently controlled to provide desired beam current at the halls.

For larger bunch time intervals, a drive laser can be operated at subharmonic frequencies of $499 \mathrm{MHz}$, for example, 249.5 and $124.75 \mathrm{MHz}$, to produce 4 and $8 \mathrm{~ns}$ intervals, but laser hardware issues make it difficult to directly extend this range. For example, it is difficult to obtain symmetric and narrow optical pulses via gain switching at frequencies below $\sim 100 \mathrm{MH} z$ and expensive pulse-picker systems and/or long laser cavity lengths are required when the technique of modelocking is used. These limitations can be overcome by operating the laser at frequencies near $499 \mathrm{MHz}$ and passing only some of the 

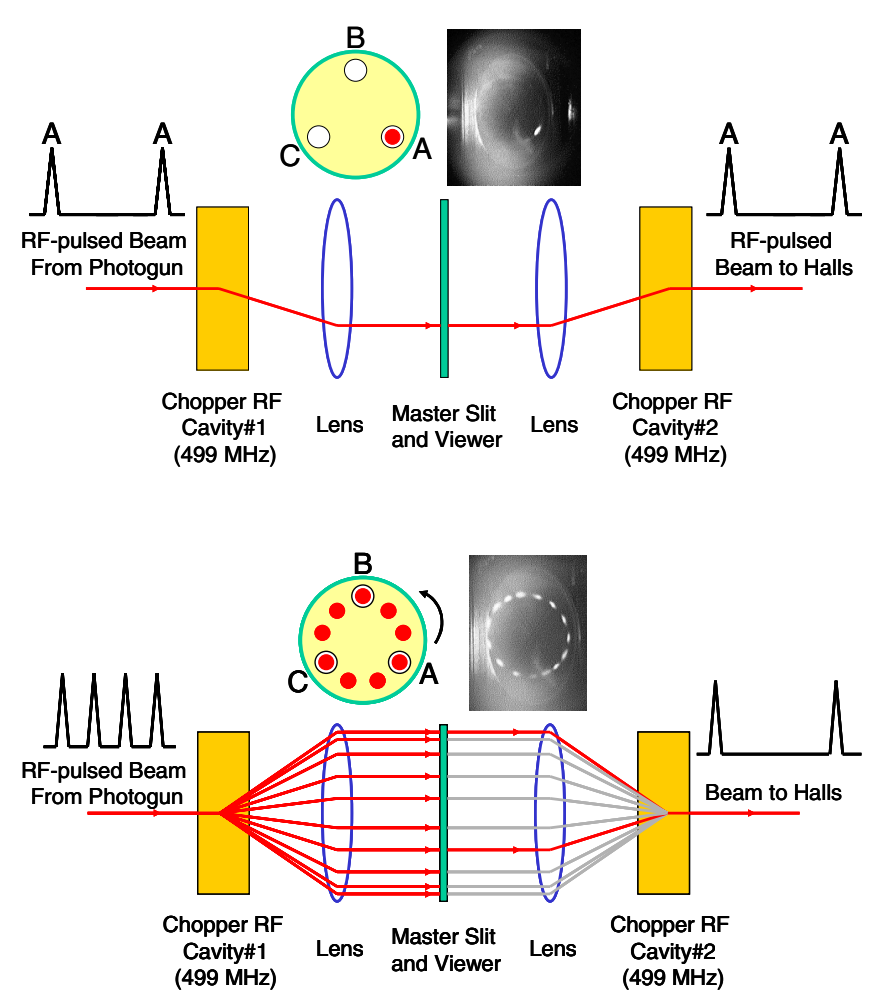

FIG. 1. (Color) Schematic representation of the CEBAF photoinjector chopper system for two different drive laser configurations: Top: "Normal" electron beam produced by one drive laser with $499 \mathrm{MHz}$ pulse repetition rate. The first rf deflecting cavity sweeps the beam across an aperture in a water-cooled copper plate ("master slit") and the second rf cavity restores the original beam trajectory. The inset shows an actual beam spot on a viewscreen located midway between the two rf cavities. Often, there are three drive lasers producing interleaved $499 \mathrm{MHz}$ pulsetrains. Each beam passes through a corresponding aperture at the master slit. Bottom: The beat-frequency technique, with one laser producing beam at $467.8125 \mathrm{MHz}$ beam. Most of the bunches are dumped at the chopper master slit. Only one of 15 bunches is delivered to an experimental hall.

electron bunches that are extracted from the gun (Fig. 1, bottom). The first of the equations below describes the relationship between the chopper and laser frequencies ( $F_{\text {chopper }}$ and $\left.F_{\text {laser }}\right)$ necessary to obtain a periodic pulse train downstream of the photoinjector chopper, where $N$ is an integer greater than one. The second equation provides the corresponding laser frequency and the third equation indicates the repetition rate for bunches delivered to the experimental hall:

$$
\begin{gathered}
\left|\frac{1}{F_{\text {chopper }}}-\frac{1}{F_{\text {laser }}}\right|=\frac{N}{F_{\text {chopper }}} \\
F_{\text {laser }}=F_{\text {chopper }}\left(\frac{N-1}{N}\right)
\end{gathered}
$$

$$
F_{\text {bunch }}=F_{\text {chopper }}-F_{\text {laser }}=\frac{F_{\text {chopper }}}{N} .
$$

Measurements were made using a new drive laser [5] that consists of a fiber-coupled, gain-switched diode seed laser and ErYb-doped fiber amplifier at $1.56 \mu \mathrm{m}$ wavelength, followed by a periodically poled lithium niobate crystal to obtain useful light at $780 \mathrm{~nm}$ via frequency doubling (Fig. 2, top). This laser is ideally suited for implementing the beat-frequency technique because the pulse forming mechanism, gain switching, does not depend on laser cavity length as does modelocking. Picosecond pulses can be obtained over a wide range of frequencies by merely changing the frequency of the rf that is applied to the seed laser.

Initial tests to demonstrate the feasibility of the method were performed using a portable rf signal generator (Hewlett-Packard Model 8662) in place of the stabilized rf master oscillator normally used to operate the photogun drive laser. Only integer frequencies were used because the rf source could not accommodate frequencies with run-on decimals. These frequencies are shown in Table I. The laser pulse frequency was varied between 399 and $479 \mathrm{MHz}$, to obtain bunch spacing from 10 and $50 \mathrm{ns,}$ respectively.

Although the portable rf source was adequate to demonstrate the feasibility of the beat-frequency method, significant timing jitter could be observed on the beam. Greatly improved beam quality was obtained using the frequency divider and mixer circuit shown in Fig. 2 (bottom). The $499 \mathrm{MHz}$ accelerator master oscillator (MO) signal was divided by integer $N=16$ using an emitter coupled logic prescaler to produce the desired low frequency bunch repetition rate. This signal was then remixed with the $499 \mathrm{MHz}$ MO to produce the proper beatfrequency sideband which was then filtered, amplified, and applied to the drive laser. This versatile approach was easy to implement and ensured phase coherence between the atypical laser frequency and the chopper frequency at $499 \mathrm{MHz}$ (after $N$ cycles), as well as maintaining the long-term timing stability. In addition, employing a low-noise, programmable divider such as an Analog

TABLE I. A list of demonstrated laser frequencies that provided "beat-frequency" beam downstream of the CEBAF photoinjector chopper.

\begin{tabular}{cccc}
\hline \hline Integer & $\begin{array}{c}\text { Laser frequency } \\
(\mathrm{MHz})\end{array}$ & $\begin{array}{c}\text { Bunch spacing } \\
(\mathrm{nsec})\end{array}$ & $\begin{array}{c}\text { Bunch } \\
\text { frequency } \\
(\mathrm{MHz})\end{array}$ \\
\hline 5 & 399.20 & 10.0 & 99.80 \\
8 & 436.625 & 16.0 & 62.375 \\
10 & 449.10 & 20.0 & 49.90 \\
16 & 467.8125 & 32.1 & 31.1875 \\
20 & 474.05 & 40.1 & 24.95 \\
25 & 479.04 & 50.1 & 19.96 \\
\hline \hline
\end{tabular}



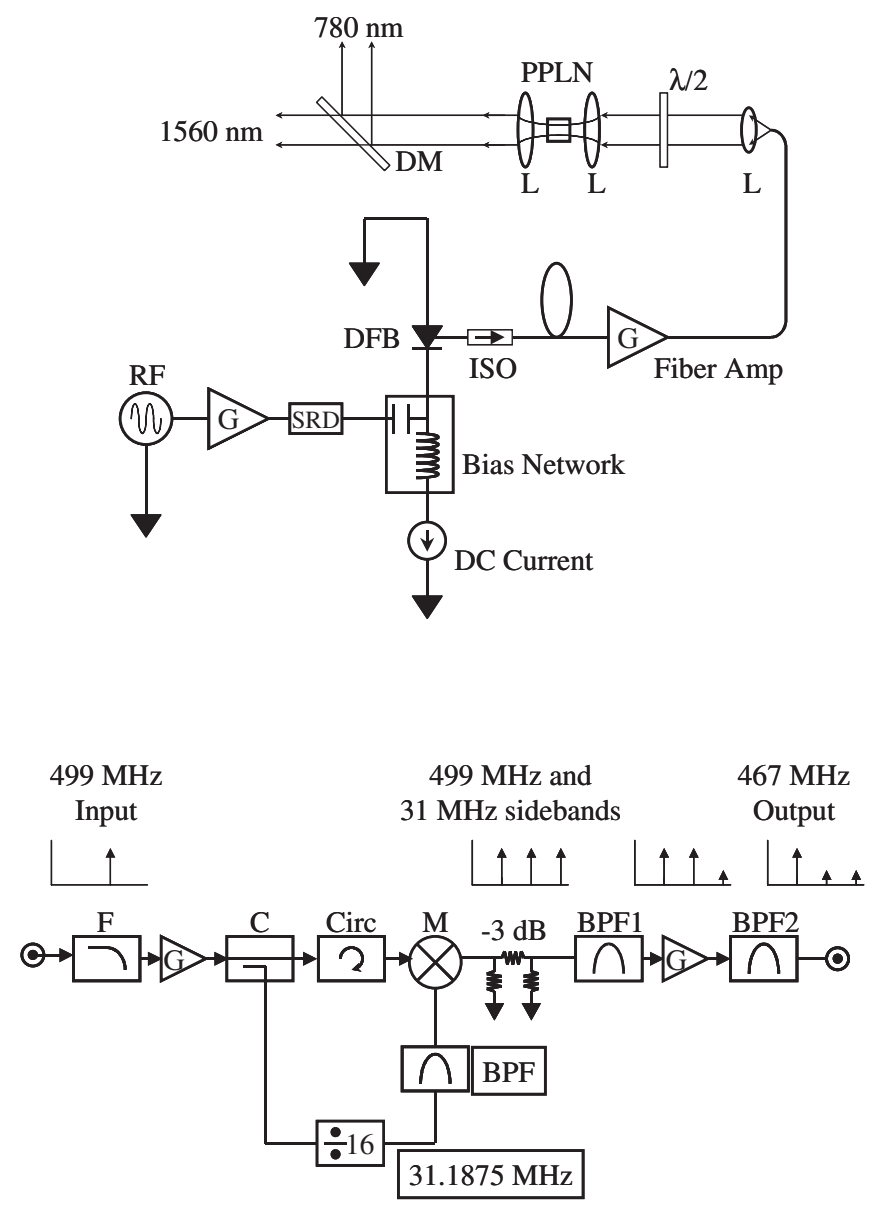

FIG. 2. Top: Schematic of the fiber-based laser system. DFB, distributed feedback Bragg reflector diode laser; ISO, fiber isolator; SRD, step recovery diode; L, lens; PPLN, periodically poled lithium niobate frequency doubling crystal; DM, dichroic mirror. Bottom: Schematic of the circuit used to generate the 467.8125 MHz signal applied to the fiber-based drive laser. F, cutoff filter; G, amplifier; C, coupler; Circ, circulator; M, mixer; BPF, bandpass filter; BPF1 and BPF2 combine to form a composite bandpass filter near $467 \mathrm{MHz}$, rejecting $499 \mathrm{MHz}$ and the upper sideband at $530.1875 \mathrm{MHz}$.

Devices AD9511 facilitates any bunch spacing in $2 \mathrm{~ns}$ increments, even frequencies with run-on decimal.

\section{RESULTS}

In "beat-frequency" mode, most of the extracted beam is dumped at the photoinjector chopper, as illustrated in Fig. 1, bottom, which depicts operation with one laser at 467.8125 MHz. For this case, there are 15 spots on the chopper viewer (inset photograph) and only one of the 15 bunches would pass through a chopper aperture, producing a beam with $32 \mathrm{~ns}$ bunch spacing delivered to an experimental hall. Transmission from the gun to the hall would be modest, only $7 \%$, because 14 out of 15 electron bunches would be dumped on the chopper aperture plate. Fur- thermore, it must be pointed out that, as the laser pulse repetition rate approaches the chopper frequency, neighboring pulses on the chopper viewer begin to overlap. When this happens, the low repetition rate beam downstream of the photoinjector chopper becomes contaminated by a high repetition rate beam at the laser frequency, a condition that is unacceptable for many applications.

The upper limit on the bunch spacing is set by the electron bunchlength at the chopper, a quantity that depends on the laser optical pulsewidth ( $\sim 40 \mathrm{ps}$, Ref. [5]), and on the beam current; electron bunches expand while traversing the distance between the gun and the photoinjector chopper due to space-charge effects, or Coulomb repulsion of the electrons within the bunch, and this is more severe at higher current. Electron bunchlength measurements were performed using the photoinjector chopper and normal electron beam at $499 \mathrm{MHz}$. For these measurements, two of the three chopper slits were closed and the remaining slit width was adjusted to pass only a small portion of the electron bunch within an $18 \mathrm{ps}$ window. The laser phase was varied to move the electron bunch across the narrow chopper slit and transmitted beam current was monitored with a downstream Faraday cup. Results are plotted in the center of each graph in Fig. 3 (left). These data were reproduced alongside actual bunches using a computer data analysis program, displaced in time with offsets appropriate for different laser repetition rates corresponding to bunch spacing of 20,31, and $50 \mathrm{~ns}$ (top to bottom), to predict beam current and beam quality during beat-frequency mode. Beam within the vertical lines on the plots (representing the width of the fully open chopper slit) will be delivered to the halls; beam outside the vertical lines would be dumped. The plots on the right side of Fig. 3 provide an estimate of high frequency contamination at the laser repetition rate and indicate that it should be possible to provide a few microamperes beam current with high frequency contamination $<1 \%$ for bunch spacing less than $\sim 30 \mathrm{~ns}$. For longer bunch intervals, high frequency contamination becomes very large (see $50 \mathrm{~ns}$, for example). Note, however, that contamination can be reduced at the expense of delivered beam current by reducing the width of the chopper slit.

\section{A. Application example: A study of Møller polarimeter systematic errors}

Electron beam polarization measurements that rely on Møller scattering are performed at low current $(<$ few $\mu \mathrm{A}$ ) to avoid target heating and subsequent target depolarization, while nuclear physics experiments are typically conducted at significantly higher current. This inconsistency between beam current conditions has been the source of concern for some nuclear physics experimenters who would rather measure polarization at the same beam 

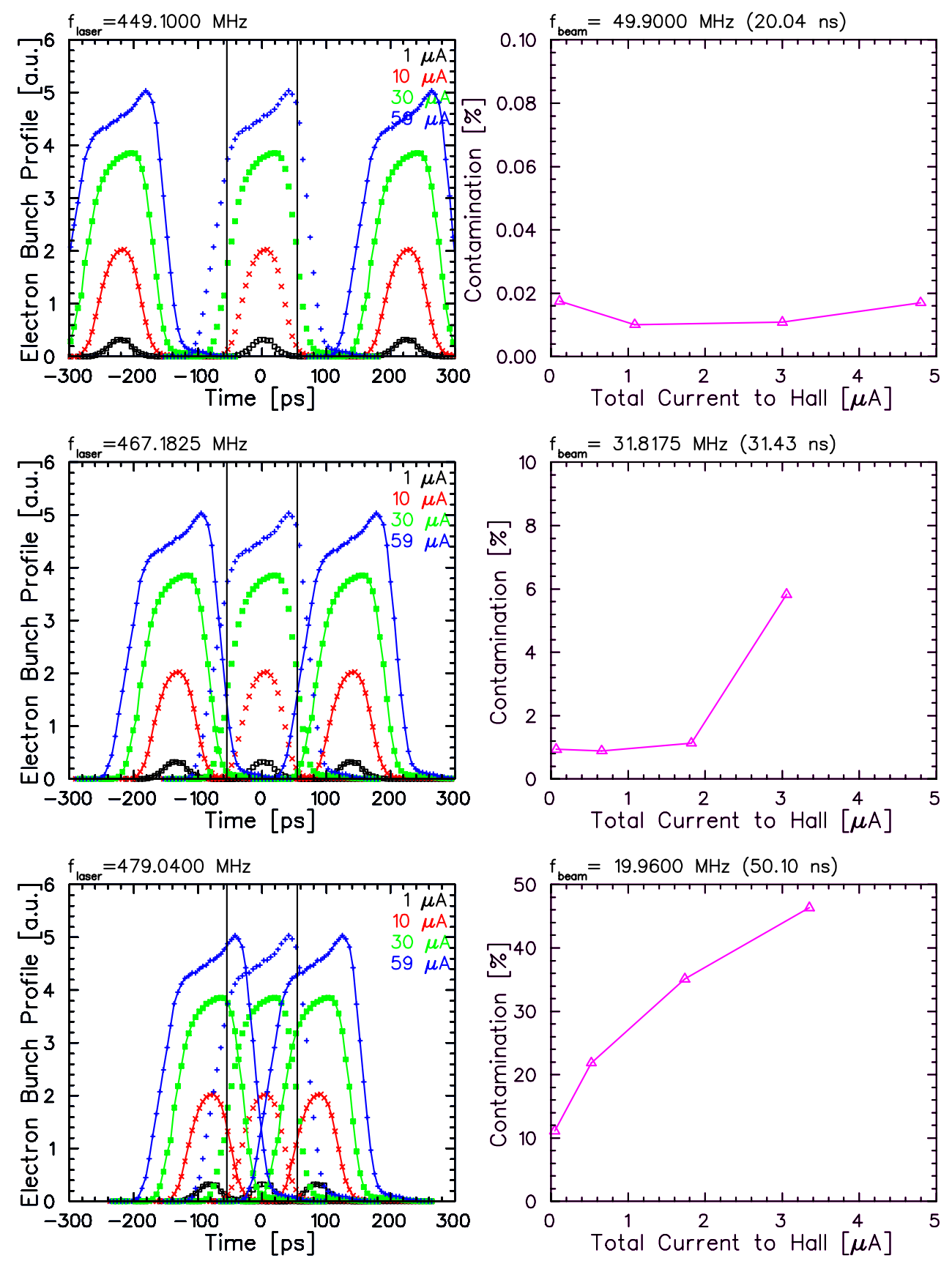

FIG. 3. (Color) Left: Electron bunchlength scans at four different gun currents and three different laser pulse repetition rates corresponding to bunch spacing of 20,31 , and $50 \mathrm{~ns}$. Beam within the vertical lines will be delivered to the experimental hall. These plots illustrate how contamination at the laser repetition rate is introduced onto the beam, a result of space-charge induced bunch lengthening. Right: Estimates of high frequency contamination as a function of delivered beam current to the experimental hall. Details are described in the text.

current as required by the experiment, to avoid systematic errors that might arise, for example, due to possible current-dependent photocathode phenomena.

In addition, Hartmann et al. [6] determined that beam polarization can vary across the temporal profile of a narrow electron bunch (few ps). Specifically, the leading edge of the pulse can have higher beam polarization compared to the trailing edge, because electrons depolarize as they diffuse to the surface of the GaAs crystal. This behavior has been observed at CEBAF, although under normal experimental conditions, nearly the entire electron bunch is delivered to the physics target and variations in polarization associated with different electron diffusion times are averaged out; experiments receive beam polarization representative of the average of all of the electrons within the bunch [7]. Problems can arise during polarim- 
etry, however, when a narrow chopper slit is used to reduce beam current to an acceptable level [8]. A narrow chopper slit passes only a small portion of the electron bunch. Chopper-slit misalignment or operation with an incorrect laser phase results in delivery of electrons from the leading or trailing edge of the bunch. As a result, the experimenters measure beam polarization that is higher or lower than actual values associated with production running.

The beat-frequency technique was employed to study the magnitude of the systematic errors described above. While using the beat-frequency technique, extracted beam current from the photogun remains high and consistent with levels used during the experiment, but delivered beam current is low because only some of the extracted electron pulses pass the chopper. Moreover, the chopper slit remains open and the entire bunch is delivered to the
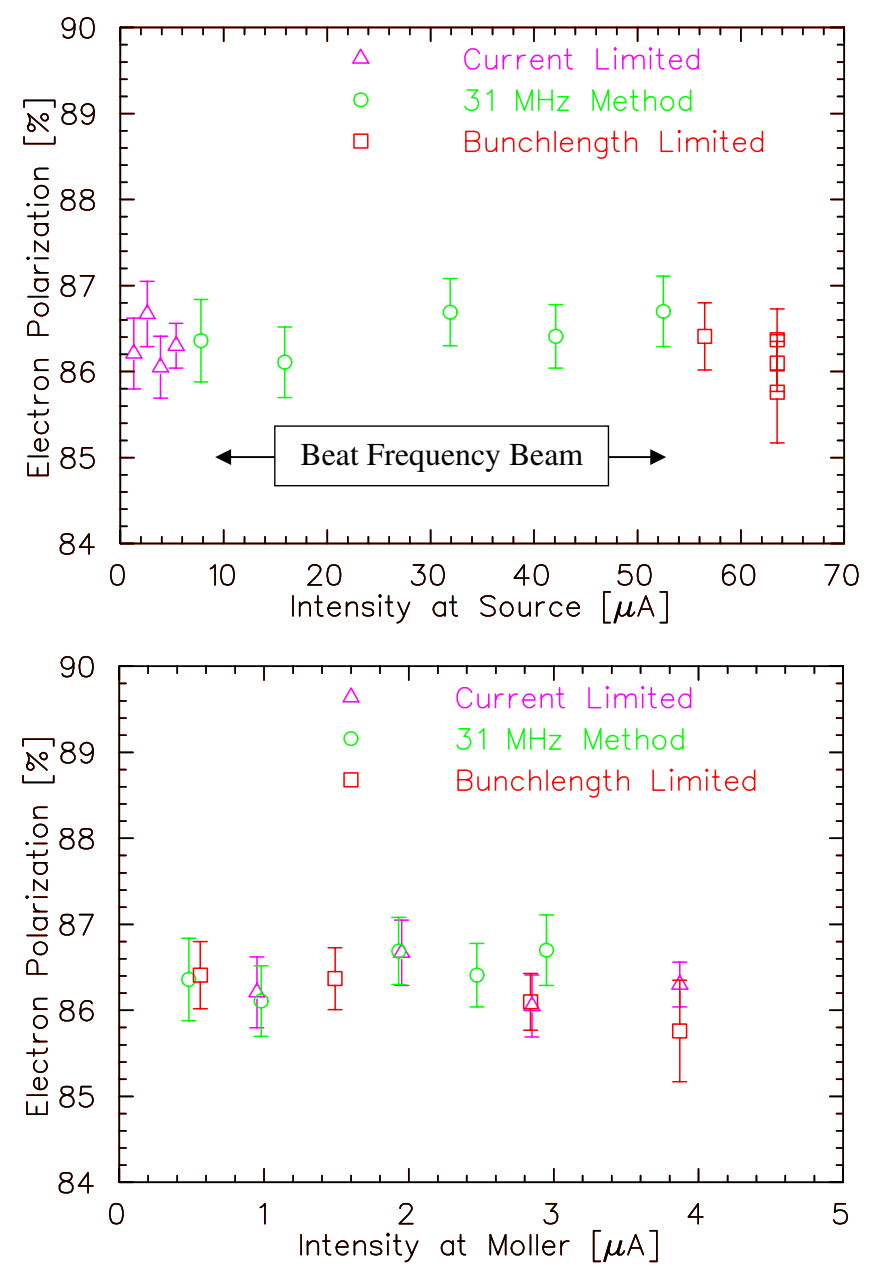

FIG. 4. (Color) Top: Electron beam polarization measured using the Hall $\mathrm{C}$ Møller polarimeter versus extracted beam current, using three different photoinjector configurations: $(\triangle)$ low current and sampling the entire bunch profile, $(\bigcirc)$ high current but selecting only a narrow portion of the electron bunch, and ( $\square$ ) high current and sampling the entire bunch via the beatfrequency technique. Bottom: The same data replotted versus average beam current at the Møller target foil. polarimeter, eliminating the possibility that beam polarization can be sensitive to different electron diffusion times within the photocathode.

Polarization measurements were performed using the Møller polarimeter [9,10] at experimental Hall C [11], for three different photoinjector configurations. First, polarization measurements were made using normal $499 \mathrm{MHz}$ beam, with low current $(<4 \mu \mathrm{A})$ extracted from the gun and nearly all the electron beam delivered to the hall through a fully open chopper slit. Next, high current was extracted from the gun $(\sim 60 \mu \mathrm{A})$ and the chopper-slit width was reduced to sample only a small portion of the electron bunch, to maintain low current and manageable background at the polarimeter. Finally, the beat-frequency technique was used to sample nearly the entire electron bunch at beam currents to $60 \mu \mathrm{A}$. Beam current at the polarimeter was low because most of the bunches (i.e., 14 out of 15) were dumped at the chopper. Results are plotted in Fig. 4, both as a function of extracted beam current from the photogun (top) and as a function of delivered beam current to the Møller target (bottom). Since polarization was high $(\sim 86 \%)$ and constant within the statistical uncertainties of the measurement $(\sim 1 \%)$, the top plot suggests systematic errors associated with the mechanisms described above are not obviously problematic. The bottom plot hints a slightly decreasing polarization that might be related to Møller target heating issues. Further measurements like these will help improve the precision of polarimetry at the Lab, an important requirement to successfully complete demanding experiments that have been conditionally approved for beamtime in the future.

\section{CONCLUSIONS}

The beat-frequency technique provides a simple way to create an electron beam with long time intervals between bunches, a highly useful tool for nuclear physics experimenters at CEBAF. Already, this beam has been delivered to two experimental halls to study sources of polarimeter systematic error (described above), to investigate sources of unwanted background, particle identification, and for detector calibration. The versatile drive lasers and the sophisticated injector chopper system make this possible. One obvious drawback of the beat-frequency technique is that most of the extracted beam gets dumped on the chopper aperture. The second drawback is that at high current, electron-bunch lengthening due to Coulomb repulsion introduces contamination from neighboring electron bunches, limiting the average beam current to a few microamperes. Despite these limitations, the method has proved to be a very useful tool in experimental data analysis and it is expected that future experiments at CEBAF will routinely use this additional feature of the polarized photoinjector. 


\section{ACKNOWLEDGMENTS}

The authors thank the $\mathrm{G}^{0}$ and $\mathrm{GE}_{n}$ collaborations, in particular, Tanja Horn, Dave Gaskell, Bogdan Wojtsekhowski, and Robert Feuerbach, for assessing beam quality at the experimental halls during the development of this technique. This paper was authored by The Southeastern Universities Research Association, Inc. under U.S. DOE Contract No. DE-AC05-84ER40150.

[1] C. K. Sinclair, P. A. Adderley, B. M. Dunham, J.C. Hansknecht, P. Hartmann, M. Poelker, J. S. Price, P. M. Rutt, W. J. Schneider, and M. Steigerwald, Phys. Rev. ST Accel. Beams 10, 023501 (2007).

[2] C. W. Leemann et al., Annu. Rev. Nucl. Part. Sci. 51, 413 (2001).

[3] D.S. Armstrong et al., Phys. Rev. Lett. 95, 092001 (2005).

[4] R. Abbott, S. Benson, M. Crofford, D. Douglas, R. Gonzales, R. Kazimi, D. Kehne, G. A. Krafft, P. Liger, H. Liu, D. Machie, and C. Sinclair, Proceedings of the 1994 International LINAC Conference, Tsukuba, Japan, 1994, edited by K. Takata, Y. Yamzaki, and K. Nakahara.

[5] J. Hansknecht and M. Poelker, Phys. Rev. ST Accel. Beams 9, 063501 (2006).

[6] P. Hartmann, J. Bermuth, J. Hoffmann, S. Kobis, H. G. Andresen, K. Aulenbacher, P. Drescher, H. Euteneuer, H. Fischer, K. Grimm, Th. Hammel, D. V. Harrach, H. Hofmann, K. -H. Kaiser, E. -M. Kabuss, H. J. Kreidel, A. Lopes-Ginja, F. E. Maas, Ch. Nachtigall, S. Plutzer, E. Reichert, M. Schemies, E. Schillig, K. H. Steffens, M. Steigerwald, and H. Trautner, Proceedings of the Workshop on Polarized Electrons and Low-Energy Polarimeters, Amsterdam, 1996; published in the Proceedings of the 12th International Symposium on
High Energy Spin Physics (SPIN96), edited by C. W. de Jager et al. (World Scientific Publishing Co., Singapore, 1997); also P. Hartmann et al., Nucl. Instrum. Methods Phys. Res., Sect. A 379, 15 (1996).

[7] From Møller polarimeter studies at experimental Hall A and communication with Eugene Chudakov, http:// www.jlab.org/ moller/laser_phase.html.

[8] The motivation for operating the photoinjector using a narrow chopper slit is described below. There are three independent drive lasers at CEBAF, one laser for each experimental hall. Each drive laser produces a small amount of DC light, and consequently, a small amount of electron beam for neighboring halls. This is problematic because the light from each drive laser does not necessarily have the same wavelength or polarization. As a result, the DC beam created by one laser can have opposite or greatly reduced beam polarization compared to that created by another drive laser. This leads to dilution of beam polarization at individual halls, a condition most evident at halls receiving low current, for example, during Møller polarimeter measurements. Polarization dilution can be minimized by extracting a large amount of current from the photogun and using the chopper attenuator slit to reduce beam current to a manageable level. In this manner, the portion of the delivered beam from other drive lasers is less than $1 \%$.

[9] M. Hauger, A. Honegger, J. Jourdan, G. Kubon, T. Petitjean, D. Rohe, I. Sick, G. Warren, H. Wöhrle, J. Zhao, R. Ent, J. Mitchell, D. Crabb, A. Tobias, M. Zeier, and B. Zihlmann, Nucl. Instrum. Methods Phys. Res., Sect. A 462, 382 (2001).

[10] L. J. deBever, M. Loppacher, J. Zhao, W. A. Tobias, and B. Zihlmann, in the Proceedings of the 12th International Symposium on High Energy Spin Physics (SPIN96) (Ref. [6]).

[11] See, for example, M.E. Christy et al., Phys. Rev. C 70, 015206 (2004). 